\section{MR HEAD RESPONSE FROM ARRAYS OF LITHOGRAPHICALLY PATTERNED PERPENDICULAR NICKEL COLUMNS}

S. Y. Yamamoto, R. O'Barr, and S. Schultz

Center for Magnetic Recording Research and Department of Physics,

University of California, San Diego, 9500 Gilman Drive, La Jolla, California $92093-0319$

$$
\text { A. Scherer }
$$

Department of Electrical Engineering, California Institute of Technology, Pasadena, CA 91125

\section{Overview}

In an effort to explore the feasibility of a patterned perpendicular magnetic storage medium, we have lithographically prepared arrays of nickel columns embedded in $\mathrm{SiO}_{2}$. The arrays have been planarized using a chemical mechanical polish. By raster-scanning a magnetoresistive (MR) head in contact with the medium, we have obtained magnetic images of the arrays, demonstrating the ability to "read" the magnetization state of the Ni columns.

Preparation method

The principle steps in the preparation of the $\mathrm{Ni}$ columns are e-beam lithography, ion milling, reactive ion etching, Ni electroplating, and chemical mechanical polish [1]. Electron beam lithography is used to write a pattern of dots in a $370 \mathrm{~nm}$ thick layer of $4 \%$ polymethyl methacrylate (PMMA). The patterns are developed in a 3:7 cellulose:methanol mixture, and then transferred to an underlying $100 \mathrm{~nm}$ Au layer by ion beam milling. The Au layer is used as a mask to transfer the patterns into a $400 \mathrm{~nm}$ thick $\mathrm{SiO}_{2}$ layer by reactive ion etching in $\mathrm{C}_{2} \mathrm{~F}_{6}$. The result is a $\mathrm{SiO}_{2}$ layer with a pattern of cylindrical holes which are then filled with $\mathrm{Ni}$ by electroplating at constant current. Typically, the nickel overplates and "mushrooms" onto the surface of the $\mathrm{SiO}_{2}$. These $\mathrm{Ni}$ bumps are removed from the surface via a chemical mechanical polish using colloidal silica. Fig. 1 shows an SEM micrograph of an unpolished sample of Ni columns with a nominal diameter of $150 \mathrm{~nm}$ and a center-to-center spacing of $2 \mu \mathrm{m}$

MR head response

"Scanning Magnetoresistance Microscopy" (SMRM) [2] has been used to image the Ni arrays. This technique is essentially a scanned probe microscopy which uses commercial MR heads as sense probes. The head is raster-scanned in contact with a medium, and a magnetic image is constructed by plotting the MR voltage as a function of scan position. Fig. 2 is a SMRM image of an array of Ni columns after chemical mechanical polish. Before polishing, attempts to image this array by SMRM were unsuccessful due to the large head/sample spacing. Because the columns have a spacing of $2 \mu \mathrm{m}$ and the MR element has a stripe width of $4 \mu \mathrm{m}$, the MR head signal on average includes signal from two adjacent columns. Since the peak signal over two columns is $50 \mu \mathrm{V}$, and since the head has a linear response, we would expect the signal from a single column to be $\sim 25 \mu \mathrm{V}$, much larger than our instrument noise floor of $\sim 1 \mu \mathrm{V}$.

Conclusions

We have imaged arrays of Ni columns with the SMRM. The MR head response demonstrates the feasibility of reading individual columns even with conventional MR heads. A high permeability "keeper", and further advances in head technology such as GMR and spin-valve heads would enable the investigation of higher density arrays with good signal-to-noise. Our demonstrated

\section{Dr. Sheldon Schultz}

UCSD, Physics, 0319

9500 Gilman Drive

el. (619) 534-4078

La Jolla, California 92093-0319 U.S.A._e-mail: sschultz@ucsd.edu ability to "read" these columns with an MR head is an important stepping stone toward the goal of using MR read/inductive write heads to switch (i.e., "record") individual columns. Acknowledgment

This work is supported by CMRR, NSF (MRSEC) DMR-94-00439, and CA Trade \& Com. Def. Conv. Matching Grant C94-0178.

[1] R. O'Barr, S.Y. Yamamoto, S. Schultz, W. Xu, and A. Scherer, Submitted to MMM ‘96. [2] S.Y. Yamamoto and S. Schultz, Appl. Phys. Lett. 69(21), 18 November 1996.

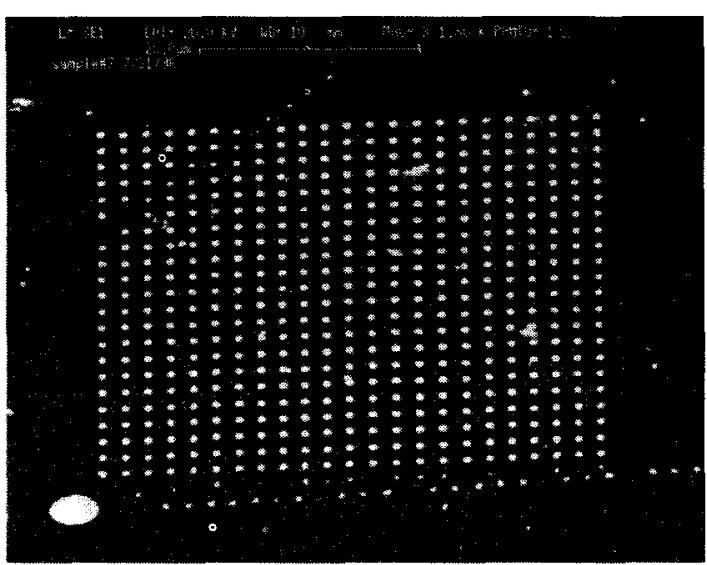

Fig. 1: SEM micrograph of an unpolished array of $\mathrm{Ni}$ columns embedded in $\mathrm{SiO}_{2}$. The columns have a nominal diameter of 150 $\mathrm{nm}$, but appear larger due to

"mushrooming" of the overplated nickel. The image is taken at a 45 degree tilt angle, and thus the array appears rectangular rather than square. The large disk at the lower left, the horizontal line at the bottom, and the $45^{\circ}$. line at the upper left of the array are all artifacts of the e-beam writing.

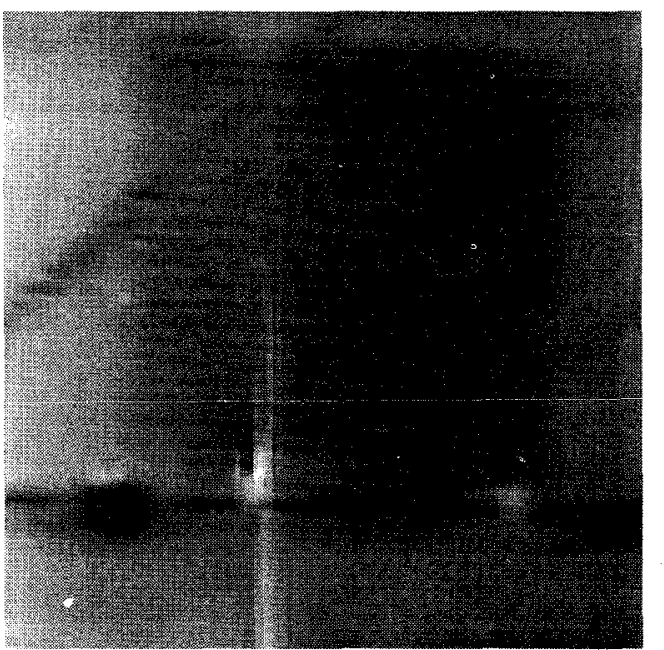

Fig. 2: A $70 \times 70 \mu \mathrm{m}$ magnetic image of a polished array of Ni columns embedded in $\mathrm{SiO}_{2}$, similar to that shown in Fig. 1. This image was recorded using Scanning Magnetoresistance Microscopy (SMRM) in which a MR head is raster-scanned in contact with the sample. Individual columns are $400 \mathrm{~nm}$ tall, $150 \mathrm{~nm}$ diameter, and spaced $2 \mu \mathrm{m}$ apart. The e-beam writing artifacts visible in Fig. 1 are clearly visible in this magnetic image. 